\title{
Design of a compact setup to generate and test optical vortex beams
}

\section{Diseño de un dispositivo compacto para generar y testear vórtices ópticos}

\author{
D. O. Pabon, S. A. Ledesma*, G. F. Quinteiro, M. G. Capeluto \\ Departamento de Física, Facultad de Ciencias Exactas y Naturales, Universidad de Buenos Aires and IFIBA, \\ CONICET, Cuidad Universitaria, Buenos Aires 1428, Argentina \\ (*) E-mail: ledesma@df.uba.ar \\ Received: 01/12/2016 Accepted: 08/08/2017 \\ DOI: $10.7149 /$ OPA.50.349030
}

\begin{abstract}
:
We present a compact design to generate and test different vortex beams with orbital angular momentum (OAM). The design consists of a mask that contains both, a binary amplitude hologram and a pinhole. When the mask is illuminated and the pinhole is blocked, the hologram produces a vortex beam with OAM whose intensity distribution can be observed in the far field. When the pinhole is unblocked a reference wavefront is generated which allows testing interferometrically the spatial phase distribution of the beam. We numerically simulate the experiment for two kind of beams containing OAM: Laguerre-Gaussian like vortex beams and Mathieu beams.
\end{abstract}

Key words: optical vortex; computer generated holograms; fork hologram; orbital angular momentum; Mathieu beams.

\section{RESUMEN:}

Presentamos un diseño compacto para generar y testear diferentes vórtices ópticos con momento angular orbital (OAM). El diseño consiste en una máscara que contiene un holograma binario y un pinhole. Cuando la máscara es iluminada y el pinhole se bloquea el holograma produce un vórtice óptico con OAM cuya distribución de intensidades se observa en el campo lejano. Cuando se deja pasar la luz proveniente del pinhole se genera una onda de referencia que permite testear de manera interferométrica la distribución especial de fase del haz. Mostramos simulaciones numéricas del experimento para dos clases de haces conteniendo OAM: un haz tipo Laguerre-Gauss y un haz Mathieu.

Palabras clave: vórtices ópticos; holograma generado por computadora; holograma tenedor; momento angular orbital; haces Mathieu.

\section{REFERENCES AND LINKS / REFERENCIAS Y ENLACES}

[1] L. Allen, M. W. Beijersbergen, R. J. C. Spreeuw, and J. P. Woerdman, "Orbital angular momentum of light and the transformation of Laguerre-Gaussian laser modes," Phys. Rev. A 45, 8185-8189 (1992). https://doi.org/10.1103/PhysRevA.45.8185

[2] Ł. Płociniczak, A. Popiołek-Masajada, M. Szatkowski, D. Wojnowski, "Transformation of the vortex beam in the optical vortex scanning microscope," Optics \& Laser Technology 81, 127-136 (2016). https://doi.org/10.1016/j.optlastec.2016.01.040

[3] B. Perez-Garcia, J. Francis, M. McLaren, R. I. Hernandez-Aranda, A. Forbes, T. Konrad, "Quantum computation with classical light: The Deutsch Algorithm", Phys. Lett. A 379, 1675-1680 (2015). https://doi.org/10.1016/j.physleta.2015.04.034 
[4] Y. Toda, K. Shigematsu, K. Yamane, R. Morita, "Efficient Laguerre-Gaussian mode conversion for orbital angular momentum resolved spectroscopy," Opt. Commun. 308, 147-151 (2013). https://doi.org/10.1016/j.optcom.2013.05.050

[5] Andrews, David L. Structured light and its applications: An introduction to phase-structured beams and nanoscale optical forces. Academic Press (2011).

[6] S. Chávez-Cerda, J. C. Gutiérrez-Vega, and G. H. C. New, "Elliptic vortices of electromagnetic wave fields," Opt. Lett. 26, 1803-1805 (2001).

https://doi.org/10.1364/OL.26.001803

[7] A. Bekshaev, O. Orlinska, M. Vasnetsov, "Optical vortex generation with a "fork" hologram under conditions of high-angle diffraction", Opt. Commun. 283, 2006-2016 (2010). https://doi.org/10.1016/j.optcom.2010.01.012

[8] L. Janicijevic and S. Topuzoski, "Fresnel and Fraunhofer diffraction of a Gaussian laser beam by forkshaped gratings," J. Opt. Soc. Am. A 25, 2659-2669 (2008) https://doi.org/10.1364/JOSAA.25.002659

[9] S. Li, and Z. Wang, "Generation of optical vortex based on computer-generated holographic gratings by photolithography," Appl. Phys. Lett. 103, 141110 (2013). https://doi.org/10.1063/1.4823596

[10] C. López-Mariscal, J. C. Gutiérrez-Vega. "The generation of nondiffracting beams using inexpensive computer-generated holograms." American Journal of Physics 75.1, $36-42$ (2007). https://doi.org/10.1119/1.2359001

[11] A. Vasara, J. Turunen, and A. T. Friberg. "Realization of general nondiffracting beams with computergenerated holograms." J. Opt. Soc. Am A 6.11, 1748-1754 (1989). https://doi.org/10.1364/JOSAA.6.001748

[12] J. Marín-Sáez, M.V. Collados, I. J. Sola, J. Atencia. "White-light optical vortices generated with holographic optical elements." Opt. Pura Apl 48, 129-133 (2015). https://doi.org/10.7149/OPA.48.2.129

[13] V. Yu Bazhenov, M. V. Vasnetsov, and M. S. Soskin. "Laser beams with screw dislocations in their wavefronts." Jetp Lett 52.8, 429-431 (1990).

[14] L. Allen, M. J. Padgett, and M. Babiker. "IV The orbital angular momentum of light." Progress in optics 39, 291-372 (1999). https://doi.org/10.1016/S0079-6638(08)70391-3

[15] A. G. Peele, P. J. McMahon, D. Paterson, C. Q. Tran, A. P. Mancuso, K. A. Nugent, J. P. Hayes, E. Harvey, B. Lai and I. McNulty, "Observation of an x-ray vortex," Opt. Lett. 27, 1752-1754 (2002). https://doi.org/10.1364/OL.27.001752

[16] A. G. Peele, K. A. Nugent, A. P. Mancuso, D. Paterson, I. McNulty and J. P. Hayes, "X-ray phase vortices: theory and experiment," J. Opt. Soc. Am. A 21, 1575-1584 (2004). https://doi.org/10.1364/JOSAA.21.001575

[17] B. Terhalle, A. Langner, B. Päivänranta, V. A. Guzenko, C. David, and Y. Ekinci, "Generation of extreme ultraviolet vortex beams using computer generated holograms," Opt. Lett. 36, 4143-4145 (2011) https://doi.org/10.1364/OL.36.004143

[18] N. C. Monserud, E. B. Malm, P. W. Wachulak, V. Putkaradze, G. Balakrishnan, W. Chao, E. Anderson, D. Carlton and M. C. Marconi, "Recording oscillations of sub-micron size cantilevers by extreme ultraviolet Fourier transform holography," Opt. Express 22, 4161-4167 (2014). https://doi.org/10.1364/OE.22.004161

\section{Introduction}

Laser beam shaping is the process to modify an optical beam, where the shape of the beam is given by the intensity distribution and its phase determines the characteristics of its propagation. It has been proven, since the invention of the laser, that any application that uses laser beams has its specific demands on a certain beam shape, including intensity or phase distribution, polarization, momentum, propagation properties. Among the variety of beams nowadays at our disposal, optical vortex (OV) beams [1] are of main interest for their wide range of applications as optical traps [2], quantum simulations algorithms with classical light [3] and spectroscopy [4], among others.

A vortex in an optical field is a point singularity where the phase is not defined [5]. The well-known Laguerre-Gaussian (LG) beams are solutions of the paraxial wave equation in cylindrical coordinates with 
a single phase singularity, around which the phase changes an integer multiple $\ell$ of $2 \pi$, where $\ell$ is also known as the topological charge. A vortex beam with topological charge $\ell$ carries orbital angular momentum $\ell \hbar$, with $\hbar$ the reduced Planck constant. Mathieu beams are solutions to the full wave equation in elliptical coordinates and exhibit multiple vortices, depending on its ellipticity. As well as Bessel beams (the solution of the wave equation in cylindrical coordinates) Mathieu beams are non-diffractive, which makes them very important in applications like lithography and imaging [6].

A possible way for obtaining optical vortices is based in the use of fork like holograms, as has been widely studied [7-14]. In order to test the vortices an interferometric set-up is required. In general, this is accomplished by means of interferometers having Michelson or Mach Zehnder architectures. In this work we propose a compact and simple device to generate and test beams carrying OAM, based on a mask composed by a binarized computer generated hologram (CGH) and a pinhole that can be switched on and off on demand. We show the device performance by running simulations on LG like and Mathieu beams. This device could have potential applications to the generation of vortex in the extreme ultra violet (EUV) region of the electromagnetic spectrum [15-18] where binary masks are required. With our proposal we avoid the use of extra optical elements and also we simplify the testing process as it simplifies the process of aligning amplitude division interferometers in the EUV.

\section{Device description}

An experimental set-up to implement the proposal is shown in Fig. 1. Light arriving from a laser is collimated by a system consisting of a microscope objective $(0)$, a pinhole $(\mathrm{PH})$ and a lens $\left(\mathrm{L}_{1}\right)$. The collimated beam impinges on the mask (as that schematized in Fig. 2) and a lens ( $\mathrm{L}_{3}$ ) is used to obtain the far field distribution of the light diffracted by the mask onto the CCD plane.

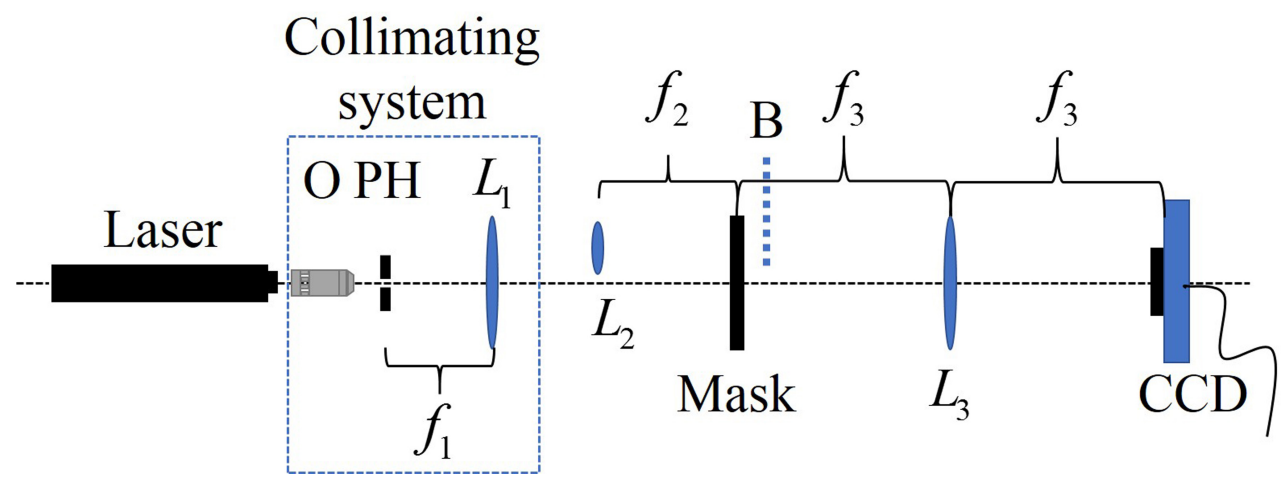

Fig. 1. The proposed experimental setup: $(\mathrm{O})$ microscope objective, $(\mathrm{PH})$ pinhole, $\left(\mathrm{L}_{1}\right)$ collimating lens, $\left(\mathrm{L}_{2}\right)$ focalizing lens, $(\mathrm{Mask})$ composed mask shown in Fig. 2, (B) depicted in dashed line represents the pinhole blocker. (L $\mathrm{L}_{3}$ ) lens with focal distance $\mathrm{f}_{3}$ and (CCD) camera for data acquisition.

The proposed mask consists of a pinhole and a laterally displaced CGH in such a way that the pinhole can be blocked or unblocked according to whether you want to generate or test the beams respectively. The blocker B is shown in dotted line in Fig. 1. In section 3 we show how to compute this kind of holograms to obtain the vortex beams.

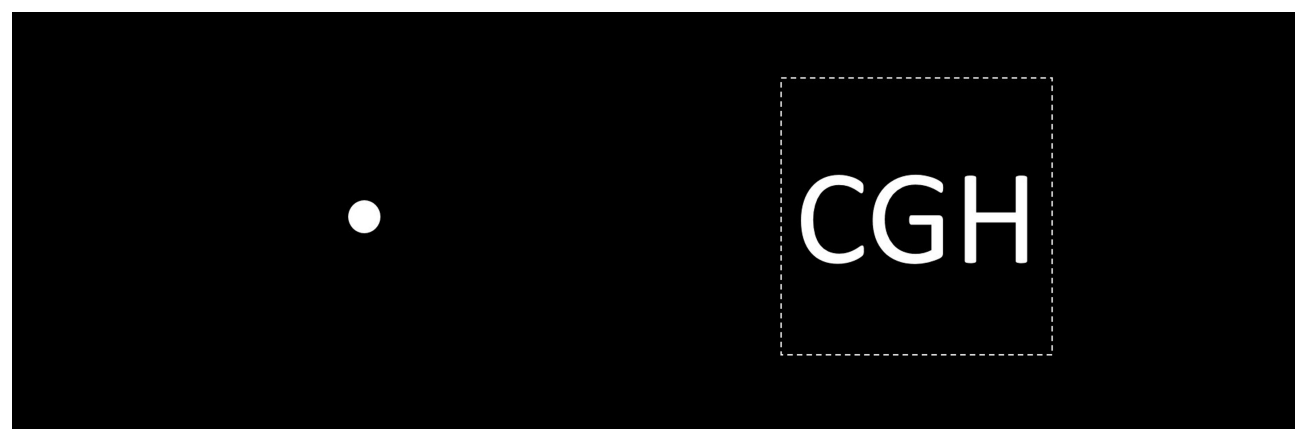

Fig. 2. Schematic of the mask composed by a pinhole and a computer generated hologram. To improve visualization the pinhole has been enlarged from its actual size. By blocking the pinhole we obtain the reconstruction of the hologram and unblocking we obtain the interference pattern of the light diffracted by the pinhole and the reconstruction of the hologram. 
When a mask like that shown in Fig. 2 is placed in the set-up depicted in Fig. 1, it is possible to generate a beam with OAM and a reference wavefront to test it. The vortex beam is generated at the far field of the hologram plane and it would be observed when the pinhole is switched off. To characterize its phase it is necessary to produce an interference pattern, this could be accomplished by unblocking the pinhole. The light diffracted from the pinhole produces at the CCD plane a plane wavefront that can interfere with the light diffracted from the CGH. As the visibility of the interference pattern depends on the intensity ratio between the light diffracted from the pinhole and that diffracted from the hologram, both intensities must be compensated for instance, by placing a lens $\left(\mathrm{L}_{2}\right.$ in Fig. 1 ) behind the pinhole for concentrating the light in this side of the mask.

\section{Simulating the generation and testing of OV}

\subsection{Binarized holograms}

In this section we show how to compute the holograms to generate LG vortex beams and Mathieu beams.

The LG vortex beams can be generated by the well-known fork holograms. They are obtained by the interference of a LG beam and a plane wave that propagates at different angle. In general, a LG beam can be expressed as:

$$
\psi_{1}(r, \varphi, z)=A_{1}(r) e^{i \ell \varphi} e^{i k_{z} z},
$$

where $A_{1}(r)$ is the amplitude distribution, $(r, \varphi)$ are polar coordinates and $k_{z}$ is the component of the wave vector in the direction $z$. The factor $e^{i \ell \varphi}$ gives the characteristic helicity to the wavefront, with a topological charge $\ell$, that indicates the number arms in the spiral phase. Fig. 3(a) shows the phase distribution (modulus $2 \pi$ ) for $\ell=1$ limited by a circular pupil. The phase at the center of the wavefront is not defined and the line indicates the change in $2 \pi$. A plane wave of amplitude $A_{2}$ that propagates in the $x z$ plane can be written as $\psi_{2}(x, z)=A_{2} e^{i k_{x} x} e^{i k_{z} z}$, where $k_{x}$ is the component of the wave vector in the direction $x$.

The interference pattern is calculated from the total electromagnetic field. The normalized intensity is given by $I=\left\{\left(A_{1}\right)^{2}+\left(A_{2}\right)^{2}+2 A_{1} A_{2} \cos \left(\ell \varphi-k_{x} x\right)\right\} /\left(A_{1}+A_{2}\right)^{2}$. In Fig. 3(b) we show an example for $\ell=1$, where the typical bifurcation at one of the groves is clearly seen. We obtained the binarized hologram by thresholding the original normalized hologram, as follows:

$$
T_{\text {bin }}= \begin{cases}1 & \text { if } \mathrm{I} \geq 0.5 \\ 0 & \text { if } \mathrm{I}<0.5\end{cases}
$$

Fig. 3(c) shows the binarization of the fork-hologram for a topological charge $\ell=1$. It should be noted that the shape of the pupil will affect the shape of the diffracted orders. Given that the CGHs for producing optical vortices are illuminated by a laser beam, in general it acts naturally as a circular limiting pupil due to its Gaussian profile. In this case, the diffracted orders produced by the hologram will have a circular geometry. Other pupil geometries will result in other shape in the diffracted orders. As in our proposal the entire mask (CGH and pinhole) must be illuminated by the beam, so to produce vortices with circular geometry we must limit the CGH with a circular pupil.

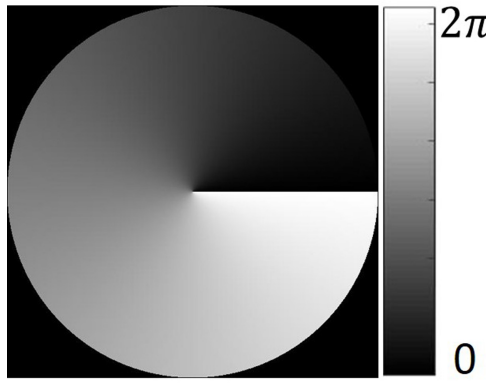

(a)

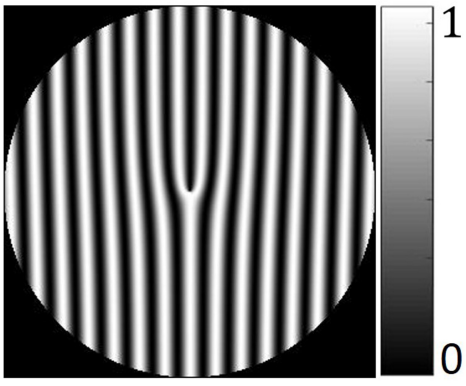

(b)

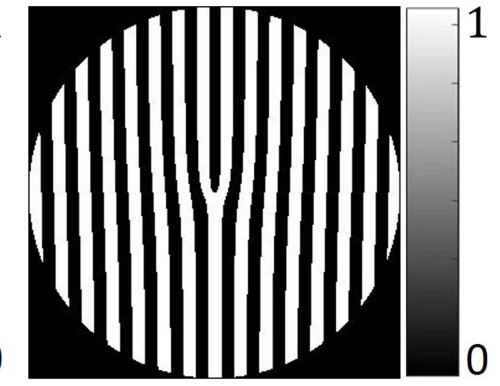

(C)

Fig. 3. (a) Distribution of the azimuthal phase given by equation (1) for a topological charge value $\ell=1$. (b) Fork hologram. (c) Binary fork hologram. The colors white and black represent the maximum and minimum values of the hologram. 
Mathieu beams are the solutions of Helmholtz's equation in elliptical coordinates $\xi$ and $\eta$, that are respectively the real and imaginary part of the $\operatorname{arcosh}[(x+i y) / f]$, with $f$ the eccentricity parameter. In these coordinates, Helmholtz's equation splits into two equations and their solutions depends on two parameters: the winding number of topological charge $(m)$ and the ellipticity parameter $(q)[6]$. The latter is related to the transverse wave vector [6]. In Fig. 4 we show the Mathieu beam for $m=6$ and $q=6$. To obtain the binarized mask to generate Mathieu beams, we proceeded as follows. First we calculated amplitude and phase of the Mathieu beam with $m=q=6$ (Fig. 4(a) and (b)). Then we Fast Fourier Transform (FFT) this complex function and obtained Fig. 4(c) and (d) for amplitude and phase respectively. The next stage was to apply an annular mask to the amplitude (Fig. 4(e)). After this, we calculated the interference pattern with a plane wave (Fig. 4(f)). At last, we binarized the interference pattern (Fig. 4(g)).

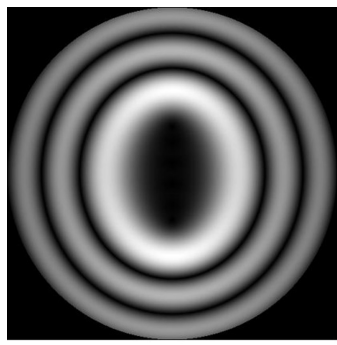

(a)

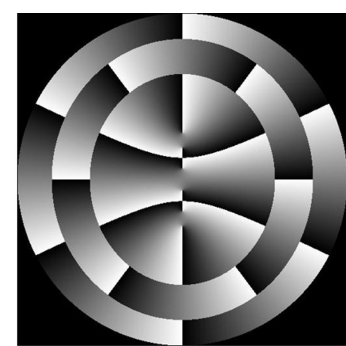

(b)

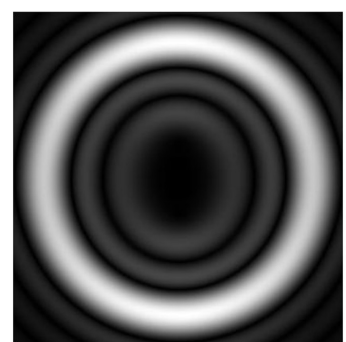

(c)

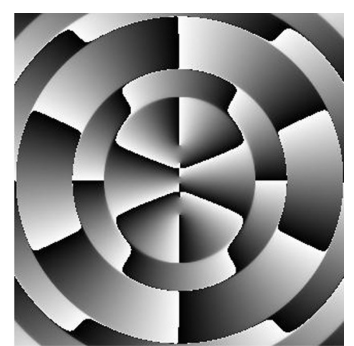

(d)

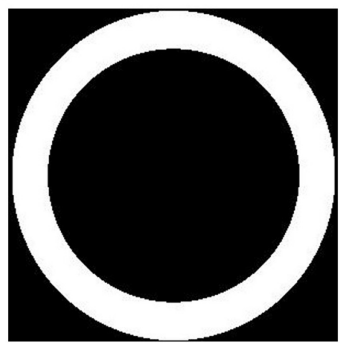

(e)

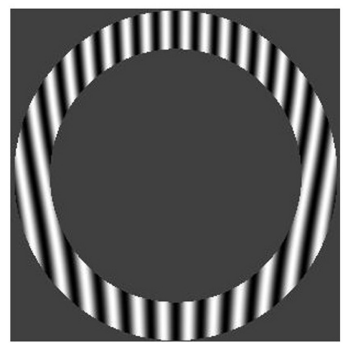

(f)

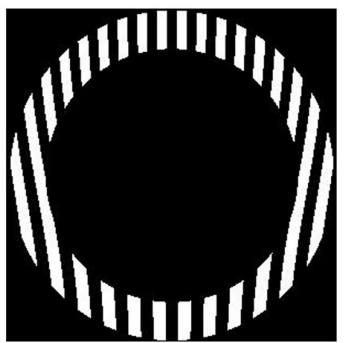

(g)

Fig. 4. Amplitude (a) and phase (b) distribution of a Mathieu beam of order and ellipticity equal to 6. (c) and (d) are respectively the amplitude and phase of the Fourier transform of the Mathieu beam. (e) binarization of (c). The hologram is calculated as the interference of a wave with amplitude (e) and phase (d) is shown in (f).

In the following section we show the simulation results for these examples.

\subsection{Amplitude and phase distribution of the beams carrying OAM}

The binarized hologram calculated above should produce a beamwhich has optical vortices. It is straightforward to observe the doughnut shaped order in the diffracted field; however, to completely characterize the wavefront it is also necessary to measure the phase. This would also allow to test if the beams are correctly generated, since for example, a failure in mask construction or misalignment could cause deviation from the desired beam. Then we used the composed mask with the CGHs shown in Fig. $3(\mathrm{c})$ and $4(\mathrm{~g})$ for producing the LG like beams or the Mathieu beams.

The LG like vortex beam would be generated in the far field of the mask, and it can be observed when the pinhole is blocked. We compute the field distribution in the far field using the FFT algorithm. The forked hologram is essentially a diffraction grating with a bifurcation in one of its grooves. Light that diffracts in this grating will produce a vortex beam in each diffraction order. As it is shown in Fig. 5(a), the intensity distribution at each diffraction order has its classical doughnut shaped profile. It has the important feature of a zero intensity in the center, that indicates the possible existence of a vortex in the propagation axis. The zero diffraction order was blocked in these figures to increase the contrast in the side diffraction orders (+/-1). 


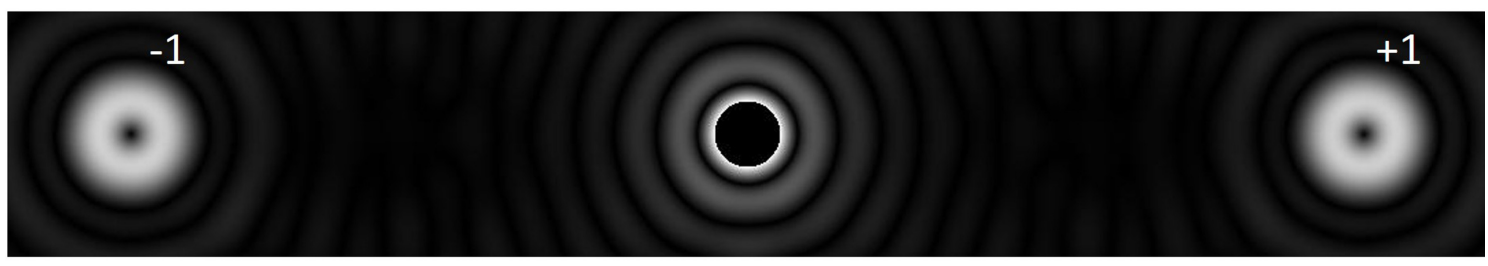

(a)

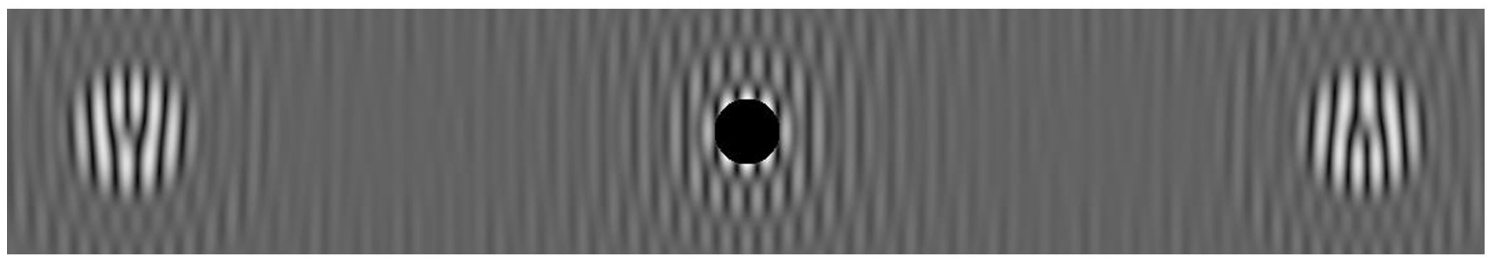

(b)

Fig. 5. (a) Simulated far field intensity distribution for a vortex beam with topological charge 1 . The zero order was blocked to be able to improved visualize the orders +1 and -1 . (b) Interference of the field distribution from (a) with the reference wavefront diffracted at the pinhole.

As it was mentioned before, by switching on the pinhole we tested interferometrically its phase. The interference of the light diffracted at the pinhole and the vortex beam, produces a forked-interference pattern at each order as it is shown in Fig. 5(b), which indicates that there is a phase singularity at the propagation axis. The number of bifurcations (in this case 1), is related to the topological charge of the beam. For our case, we have selected a pinhole of one pixel and a hologram of 300x300 pixels. Then we have maximized the visibility by multiplying the pinhole transmittance by a correction factor $(9000$ for this case).

A similar process was followed for simulating the Mathieu vortex beam. When the pinhole is blocked we obtained the intensity distribution shown in Fig. 6(a). To test the generated beam, the pinhole is switched on and the interference pattern reveals the bifurcations, typical from Mathieu beams (Fig. 6(b)). For the simulation shown in Fig. 6(c) the pinhole amplitude was multiplied by a factor of 2250 in order to maximize the visibility of the interference pattern. Although the size of the hologram is also $300 \times 300$ pixels, it is clear that the number of white pixels is less than in the previous case leading to a lower value of the multiplying correction factor.

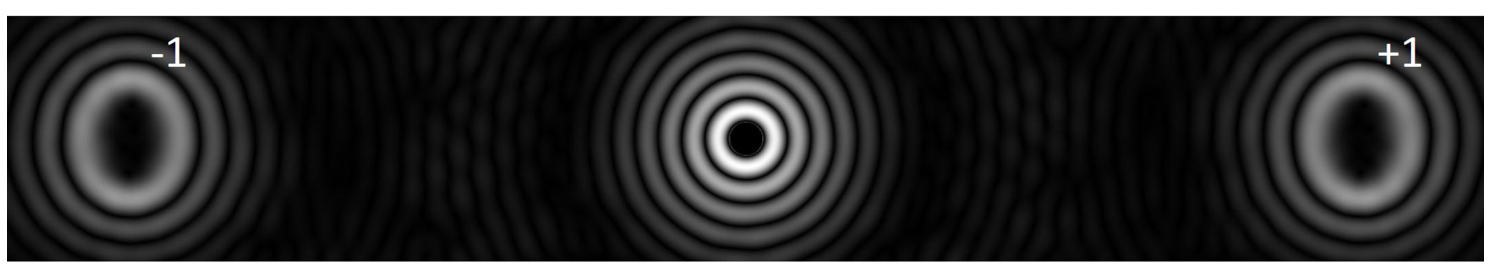

(a)

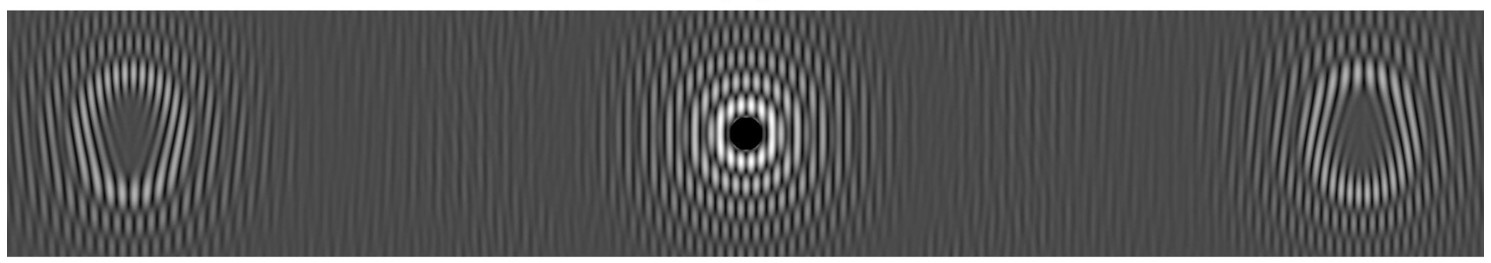

(b)

Fig. 6. (a) Simulated intensity distribution for a Mathieu beam with topological $m=q=6$. The zero order was blocked to visualize the orders +1 and -1 . (b) Interference of the field distribution from (a) with the reference wavefront diffracted by the pinhole.

\section{Conclusions}

In this work we propose a compact architecture to generate and test beams with optical angular momentum. The device consists of a binary mask, where a binary hologram and a pinhole are represented. 
In the proposed configuration, the beams with OAM are generated by blocking the pinhole, and the testing by unblocking it, which enables us to measure its phase by interference. Simulations show that the device is capable of producing and testing Laguerre Gaussian like and Mathieu beams by using binary masks. This device could have potential applications to generate vortex in the Extreme UV region of the electromagnetic spectrum where binary elements could be the only resource for shaping the beam. It should be noted that although here we have shown two examples of beams, in principle the device can serve to implement and test any type of vortices by placing in one of the halves of the mask its Fourier hologram.

\section{Acknowledgements}

The authors would like to thank the financial support from ANPCyT (Agencia Nacional de Promoción de Ciencia y Técnica): PICT-2014-3537, PICT 2014-2432, PICT 2013-592.

Portions of this work were presented at the RIAO Optilas 2016. 